\title{
IDEACIÓN SUICIDA EN ESTUDIANTES DE MEDICINA DE LA UNIVERSIDAD NACIONAL DE ASUNCIÓN
}

\author{
SUICIDAL IDEATION IN MEDICAL STUDENTS \\ OF THE NATIONAL UNIVERSITY OF ASUNCIÓN
}

Julio AMARILLA ${ }^{1}$, Fátima BARRIOS ${ }^{1}$, Fabián BOGADO ${ }^{1}$, Romel CENTURIÓN ${ }^{1}$, David CAREAGA $^{1}$, Johanna CARDOZO ${ }^{1}$, Walter GUILLÉN ${ }^{1}$, Yamila FERREIRA ${ }^{1}$, Arturo TRINIDAD ${ }^{1}$, Carol MAGGI ${ }^{2}$, Andrés ARCE ${ }^{3}$.

${ }^{1}$ Estudiantes de Medicina y Cirugía, Facultad de Ciencias Médicas, Universidad Nacional de Asunción, San Lorenzo - Paraguay

${ }^{2}$ Auxiliar de la Enseñanza de Psiquiatría, Facultad de Ciencias Médicas, Universidad Nacional de Asunción, San Lorenzo - Paraguay.

${ }^{3}$ Profesor Titular y Jefe de la Cátedra de Psiquiatría, Facultad de Ciencias Médicas, Universidad Nacional de Asunción, San Lorenzo - Paraguay.

Cómo citar este artículo: Amarilla J, Barrios F, Bogado F, Centurión R, Careaga D, Cardozo J, et al. Ideación suicida en estudiantes de Medicina de la Universidad Nacional de Asunción. Medicina Clínica y Social. 2018;2(1):13-24.

\section{RESUMEN}

Introducción: Los estudiantes de Medicina forman parte de uno de los grupos con más alto riesgo de suicidio. Objetivo: Determinar la frecuencia de ideación suicida en estudiantes de Medicina de la Universidad Nacional de Asunción. Metodología: Estudio observacional descriptivo, de corte transversal, con muestreo no probabilístico por conveniencia. Se utilizó la Escala de Ideación Suicida de Beck, adaptada al español por la Universidad Complutense de Madrid, en 288 estudiantes de Medicina de la Universidad Nacional de Asunción. Resultados: Se encontró que la frecuencia de ideación suicida fue del 54,9\%. Entre los estudiantes con ideación suicida, la intensidad del deseo de vivir fue en su mayoría de moderada a intensa (77,2\%). La intensidad del deseo de morir fue mayoritariamente débil con un $51,9 \%$. La mayoría de los pensamientos/comportamientos suicidas se presentaron de forma ocasional, rara $(62,02 \%)$ y por períodos breves y pasajeros $(81,6 \%)$. La razón más frecuente del proyecto de intento suicida fue la de escapar, acabar y resolver problemas (87,34\%). El factor disuasorio de intento de suicidio más frecuente (47\%) estuvo representado por la familia del estudiante. Discusión: La ideación suicida es un fenómeno de frecuente ocurrencia en estudiantes de Medicina. Es necesario que las Universidades establezcan programas de detección e intervención temprana que permitan disminuir la probabilidad de ocurrencia de desenlaces negativos.

Palabras clave: Ideación suicida; Suicidio; Intento de suicidio; Estudiantes de Medicina. 


\section{ABSTRACT}

Introduction: Medical students are part of one of the groups with the highest risk of suicide. Objective: To determine the frequency of suicidal ideation in medical students of the National University of Asunción. Methodology: Observational, descriptive, cross-sectional study, with non-probabilistic sampling by convenience. We used the Beck Suicidal Ideation Scale, adapted to Spanish by the Complutense University of Madrid, in 288 medical students from the National University of Asunción. Results: It was found that the frequency of suicidal ideation was $54.9 \%$. Among students with suicidal ideation, the intensity of the desire to live was mostly moderate to intense (77.2\%). The intensity of the wish to die was mostly weak with $51.9 \%$. The majority of suicidal thoughts/behaviors were occasional, rare (62.02\%) and brief and temporary (81.6\%). The most frequent reason for the suicide attempt project was to escape, finish and solve problems (87.34\%). The most frequent deterrent to attempted suicide (47\%) was represented by the student's family. Discussion: Suicidal ideation is a phenomenon of frequent occurrence in medical students. It is necessary that the Universities establish programs of detection and early intervention that allow to diminish the probability of occurrence of negative outcomes.

Keywords: Suicidal ideation; Suicide; Suicide attempt; Medical students.

\section{INTRODUCCIÓN}

El suicidio es el resultado de una compleja interacción de factores biológicos, genéticos, psicológicos, sociológicos, culturales y ambientales (1). Las conductas suicidas se han constituido en una causa importante de morbimortalidad a nivel mundial, con una incidencia anual entre 10 y 20 por 100 mil habitantes en la población general (2). La Organización Mundial de la Salud (OMS) pronostica un incremento progresivo del número de suicidios, el cual se aproximará al millón de muertes en el año 2020 (3).

Debe diferenciarse por un lado la ideación suicida, que consiste en "el pensamiento y la planeación recurrente que realiza un individuo para cometer un suicidio, pero que no llega a realizarlo en ningún momento"; por otro lado tenemos el intento suicida, que es "aquel acto que realiza un individuo, de manera voluntaria y planeada, con el objetivo de finalizar su vida"; en caso de no lograrlo queda como intento suicida fallido, pero en caso de lograr su cometido entonces hablamos de suicidio consumado (4). El suicidio es un proceso dinámico en que las anteriores conductas suelen darse en forma progresiva, de tal manera que la ideación suicida es una condición necesaria para desplegar acciones suicidas (5).

Aunque los porcentajes de suicidio varían por categoría demográfica, en los últimos 50 años han aumentado aproximadamente en un $60 \%$. Reducir la pérdida de vidas por suicidio se ha convertido en una meta de salud mental internacional fundamental $(1,6)$.

Los factores protectores reducen el riesgo de suicidio y son los siguientes: apoyo de la familia, amigos y otras personas importantes; creencias religiosas, culturales y étnicas; participación en la comunidad; vida social satisfactoria; integración social (por ejemplo, a través del trabajo, el uso constructivo del tiempo libre, etcétera); acceso a servicios y asistencia de salud mental. Aunque no eliminan el riesgo de suicidio, tales factores protectores pueden contrarrestar la extrema tensión de los acontecimientos de la vida (1).

Los comportamientos suicidas son más comunes bajo ciertas circunstancias debido a factores culturales, genéticos, psicosociales y ambientales. Los factores generales de riesgo son: bajo 
nivel socioeconómico y educativo; pérdida de empleo; tensión social; problemas con el funcionamiento de la familia, las relaciones sociales y los sistemas de apoyo; traumas, tales como abuso físico y sexual; pérdidas personales; trastornos mentales (depresión, trastornos de la personalidad, esquizofrenia, alcoholismo y abuso de sustancias, entre otros); sentimientos de falta de valor o desesperanza; problemas de discriminación por orientación sexual; comportamientos idiosincrásicos (tales como estilo cognitivo y constelación de la personalidad); problemas de juicio, falta de control de los impulsos y comportamientos autodestructivos; poca capacidad para enfrentar problemas; enfermedad física y dolor crónico: exposición al suicidio de otras personas; acceso a medios para hacerse daño; acontecimientos destructivos y violentos (tales como guerras o desastres catastróficos (1,7-10).

En general, algunos autores sostienen que la ideación suicida está conformada por cuatro etapas: el deseo de morir, la representación suicida (fantasías pasivas de suicidio), la ideación suicida sin un método determinado (idea de autodestrucción sin planteamiento de la acción) y la idea suicida planificada (suicidio planeado). Es evidente que a partir de este último factor se vuelve inminente la llegada del intento suicida que, de lograrse, se consideraría un suicidio consumado, como se mencionó previamente $(11,12)$.

El comportamiento suicida se ha incrementado en los últimos años en el Paraguay. Así lo menciona un estudio titulado "Situación actual del suicidio y tentativa de suicidio en el Paraguay", en el que se investigó retrospectivamente un universo enfocado en sujetos que cometieron suicidio e intento de suicidio en el país desde 1998 hasta 2010. Se encontraron tasas de suicidio de 4/100.000 habitantes e intentos de suicidio en 1/100.000 habitantes en el periodo 1998 - 2004 y tasas de suicidio de 5/100.000 habitantes e intentos de suicidio en 2/100.000 habitantes en el periodo 2005 - 2010. Las tasas de suicidio e intento de suicidio más elevadas, se dieron en zonas como Concepción, Amambay y Alto Paraguay. Los suicidios e intentos de suicidio se cometieron más frecuentemente entre los 18 y 29 años (13).

Es bien sabido que, en la mayor parte de las instituciones educativas de Medicina del mundo, el nivel de exigencia para el aprendizaje de conocimientos extensos y el dominio de técnicas de diagnóstico y tratamiento médico-quirúrgico hacen que los estudiantes de Medicina estén expuestos a niveles de ansiedad y estrés que pudieran ser superiores a los observados en otras disciplinas. Los estudiantes de Medicina se enfrentan a horarios de clase partidos, evaluaciones complicadas, alto nivel de competitividad, carga excesiva de trabajo, pocas horas de sueño e incertidumbre sobre la aplicación de sus conocimientos teóricos en situaciones reales y sobre sus logros a futuro $(11,12)$.

En ese sentido, y como ejemplo, en Paraguay algunos investigadores han buscado valorar la frecuencia de trastornos neuropsiquiátricos en estudiantes de Medicina, por medio de diversas escalas. Encontraron que $58,2 \%$ de la muestra presentaba algún trastorno mental, entre ellos los episodios hipomaníacos (21\%), los trastornos de ansiedad generalizada $(16,5 \%)$ y los trastornos depresivos (23,1\%). Aunque no fue la finalidad de su estudio, analizaron que una tercera parte de los estudiantes de Medicina con trastornos depresivos presentó riesgo suicida (14).

Una de las escalas de ideación suicida más frecuentemente usada es la Escala de Ideación Suicida de Beck (1979). En dicha escala se busca determinar la prevalencia de la ideación suicida a través de ítems que analizan la posibilidad de haber pensado en suicidio, la frecuencia 
de aparición de dichos pensamientos y el tiempo transcurrido desde que comenzó dicha ideación. Dicho instrumento permite determinar si un individuo presenta ideación suicida y, con ello, determinar porcentajes de frecuencia en distintas poblaciones (15).

El objetivo del presente trabajo es el de determinar la frecuencia de ideación suicida en estudiantes de Medicina de la Universidad Nacional de Asunción en el año 2017; asimismo, se buscó determinar la intensidad de los deseos de morir y vivir, describir la razón más frecuente del proyecto de intento suicida, la frecuencia en cuanto a duración y frecuencia de los pensamientos suicidas, el factor disuasorio más frecuente de un intento activo, así como, determinar la cantidad de intentos suicidas previos en la población estudiada.

Los resultados de este estudio pueden significar un gran avance en la epidemiología del suicidio en la población de estudiantes de Medicina ya que proporcionará datos útiles para futuras investigaciones a profundidad, que permitan comprender mejor esta problemática del suicidio de la que ni el personal de blanco queda exenta.

No existen estudios publicados en Paraguay sobre la frecuencia de ideación suicida en estudiantes de Medicina, por lo cual se espera que los resultados del presente estudio promuevan en las Universidades la necesidad de establecer programas de detección e intervención temprana en estudiantes en riesgo de condiciones emocionales y conductas suicidas.

\section{METODOLOGÍA}

El presente fue un estudio observacional, descriptivo de corte transversal con muestreo no probabilístico por conveniencia que se realizó de septiembre a octubre del 2017 en estudiantes de Medicina de la Universidad Nacional de Asunción. Se incluyó a todos los estudiantes que aceptaron participar voluntariamente y se excluyó a aquellos que no respondieron todas las preguntas. El tamaño de la muestra fue calculado teniendo en cuenta una frecuencia de $18 \%$ de ideación suicida (16), un intervalo de confianza del $95 \%$, un error esperado del $5 \%$ y una población de 900, quedando finalmente establecido en 182 sujetos.

Las variables sociodemográficas fueron la edad (en años cumplidos), sexo (masculino, femenino), curso $\left(1^{\circ}-6^{\circ}\right)$.

Las variables que estudian el suicidio fueron ideación suicida (sí, no); momento de la ideación (antes de ser estudiante, siendo estudiante, en ambos); deseo de vivir (ninguno, débil, moderado a intenso), deseo de morir (ninguno, débil, moderado a intenso), razones para vivir (superiores a las de morir, igual, superiores a la de morir), deseo de un intento activo de suicidio (ninguno, débil, moderado a intenso), intento pasivo de morir (dejar al azar la vida o muerte; tomar precauciones, evitar la muerte), duración del pensamiento suicida (breve o pasajero, continuo crónico o casi continuo, periodos más largos), frecuencia de pensamiento suicida (rara u ocasional, intermitente, persistente o continuo), actitud hacia el pensamiento suicida (aceptación, rechazo, ambivalencia), control sobre la acción del suicidio o deseo de llevarlo a cabo (no tiene control, tiene sensación de control, no tiene sensación de control), factores disuasorios (familia, religión, irreversibilidad, posibilidad de quedar seriamente afectado, otros), razones del proyecto de intento (escapar, acabar, resolver problemas; manipular el medio, atraer atención, venganza; ambos), factores disuasorios del intento activo (factores tienen influencia, no intentaría el suicidio debido a algún factor disuasorio, influencia mínima 
del factor), consideración de detalles en el plan suicida/métodos especificado (no los ha considerado, están detallados; los ha considerado, pero no detallado), accesibilidad/oportunidad para llevar a cabo el intento suicida (método no accesible: no hay oportunidad; el método llevaría tiempo / esfuerzo; método y oportunidad son fácilmente accesibles; oportunidades o accesibilidad futura del método proyectado), sensación de capacidad para llevar a cabo el intento de suicidio (seguro de su competencia, coraje; inseguro, competencia; no tiene coraje, demasiado débil), expectativas de un intento real de suicidio (sí, no, incierto no seguro).

Para medir las variables previamente enunciadas, se utilizó la adaptación española de la Scale for Suicide Ideation (SSI), elaborada y validada por A. T. Beck, M. Kovacs, A. Weissman (1979), con la traducción y validación hecha en la Universidad Complutense de Madrid, España (17).

Este instrumento es una entrevista semi-estructurada, autoadministrada, cuyo objetivo es el de cuantificar y evaluar la intencionalidad suicida, o grado de seriedad e intensidad con el que alguien pensó o está pensando en suicidarse, enfocada a una población de 17 años en adelante, con un consistencia interna alta (medida por un coeficiente alfa de Cronbach de 0,89$0,96)$ y una fiabilidad inter examinador de 0,83 , validada en correlación del BBS con la Escala de Desesperanza de Beck (BDI): 0,25-0,75; y con la Escala para la Evaluación de la Depresión de Hamilton (Ham-D): 0,30.

Se encuentra dividida en varias secciones que recogen una serie de características relativas a: actitud ante la vida/muerte, pensamientos o deseos suicidas, proyecto de intento de suicidio, realización del intento proyectado, y añade una quinta sección, con dos ítems en la que se indaga sobre antecedentes de intentos previos de suicidio. Estos dos ítems tienen un valor meramente descriptivo, ya que no contabilizan en la puntuación global de la escala. Para cada ítem hay tres alternativas de respuesta que indican un grado creciente de seriedad y/o intensidad de intencionalidad suicida.

La persona selecciona, para cada ítem, el nivel de intensidad/seriedad que mejor refleje las características de la ideación suicida. Si la persona contesta con un 0 ( $0=$ =ausente) a los ítems 4 y 5 , pasa directamente a contestar el ítem 20; si, por el contrario, la respuesta al ítem 4 o 5 es 1 o 2 (2=máxima intensidad), la persona debe contestar todos los ítems. Sólo se contesta el ítem 21 cuando la respuesta al ítem 20 haya sido 1 o 2 .

Cada ítem se puntúa de 0-2 ( $0=$ ausente hasta 2= máxima intensidad de la ideación suicida), y la puntuación total de la escala es la suma de los valores asignados a los 19 primeros ítems (como ya se ha indicado, los dos últimos ítems -20 y 21- tienen solo valor descriptivo).

El rango de puntuación total es de 0-38. Se considera que una puntuación igual o mayor que 1 es indicativa de riesgo de suicidio, indicando una mayor puntuación un más elevado riesgo de suicidio. Las encuestas fueron distribuidas a través de las redes sociales y enviadas por correo electrónico a los delegados de cada curso de la carrera de Medicina y Cirugía de la Facultad de Ciencias Médicas de la Universidad Nacional de Asunción. Dicho correo explicaba los objetivos del trabajo y animaba a los estudiantes a participar anónima y libremente, a través de un enlace al que podían acceder al formulario con las preguntas que fue previamente elaborado mediante la herramienta Google Forms. El cuestionario tuvo un tiempo de aplicación aproximado de 5-10 minutos. 
Para el procesamiento de la información se creó una base de datos en el software Google Sheets a partir de la cual se transfirió al software Epi Info 7.2 y Microsoft Excel 2016 en el que los datos fueron representados en medidas de tendencia central, dispersión y gráficos.

Se tomaron las medidas necesarias para respetar la privacidad de los estudiantes y la confidencialidad de la información obtenida. Quienes participaron en el presente estudio en condición de sujetos de investigación, lo hicieron de forma voluntaria y firmaron el consentimiento informado antes de la inclusión en el estudio. Por otra parte, teniendo en cuenta la potencial sensibilidad que pudiesen generar algunas de las preguntas incluidas en el cuestionario, el protocolo fue presentado y aprobado por el tutor del trabajo y por la Cátedra de Psiquiatría de la Facultad de Ciencias Médicas de la Universidad Nacional de Asunción.

Toda la información recabada fue respetada en su carácter confidencial, de privacidad, bajo anonimato y codificación numérica de las encuestas. Además, se dejaron recomendaciones y los horarios de atención de la Cátedra de Psiquiatría del Hospital de Clínicas de la Facultad de Ciencias Médicas de la Universidad Nacional de Asunción, para aquellos estudiantes interesados.

\section{RESULTADOS}

El estudio fue realizado con 288 estudiantes (del primer al sexto curso) de la carrera de Medicina de la Universidad Nacional de Asunción. Cada alumno fue sometido a una serie de preguntas en una encuesta anónima sobre ideación suicida.

\section{Variables sociodemográficas}

Con respecto a la edad se obtuvo una media de $22,79 \pm 2$, una mediana de 23 y una moda igualmente de 23. De todos los estudiantes encuestados, la mayor edad encontrada fue de 29 años y la menor edad encontrada fue de 18 años. En relación con el sexo, 195 fueron mujeres $(67,7 \%)$ y $93(32,3 \%)$ varones. Con respecto a los cursos a los que pertenecían los participantes, la mayor parte (56) fueron estudiantes del $5^{\circ}$ curso; luego siguió el $2^{\circ}$ curso con 55 participantes; en tercer lugar, se encontraron el $3^{\circ}$ y el $4^{\circ}$ curso con 48 participantes cada uno; después, el $1^{\circ}$ curso con 46 participantes; y finalmente, el $6^{\circ}$ curso con 35 participantes.

\section{Variables sobre el suicidio}

Al preguntarles a los encuestados si tuvieron alguna vez ideas suicidas en su vida, 158 personas $(54,9 \%)$ contestaron que sí; por el otro lado, $130(45,1 \%)$ contestaron que no. Con respecto a la pregunta "¿En qué momento de tu vida has tenido ideas suicidas?", 69 estudiantes $(43,7 \%)$ contestaron que antes y durante su vida universitaria; 58 estudiantes $(36,7 \%)$ respondieron siendo estudiantes universitarios; y $31(19,6 \%)$ contestaron antes de ser estudiante universitario.

El deseo de vivir fue expresado de la siguiente manera: 123 estudiantes $(77,8 \%)$ contestaron "Moderado a intenso"; 30 estudiantes (19\%) marcaron la opción "Débil"; y, por último, 5 $(3,2 \%)$ respondieron "Ninguno". Por su parte, el deseo de morir fue expresado de la siguiente manera: 82 estudiantes (51,9\%) contestaron "Débil"; 47 estudiantes (30\%) marcaron la opción "Ninguno"; y, por último, 29 (18,1\%) respondieron “Moderado a intenso". 
Con referencia a las "Razones para vivir/morir", 120 de los encuestados (75,9\%) contestaron "Las razones para vivir son superiores a las de morir"; 26 (16,5\%) dijeron que las razones para vivir como para morir son iguales; $y$, por último, 12 encuestados $(7,6 \%)$ respondieron que "Las razones para morir son superiores a las de vivir". El deseo de realizar un intento activo de suicidio fue expresado de la siguiente manera: 97 estudiantes $(61,3 \%)$ respondieron "Ninguno"; 52 estudiantes (33\%) respondieron "Débil"; y finalmente, $9(5,7 \%)$ respondieron "Moderado a intenso". El deseo de realizar un intento pasivo de suicidio se expresó de la siguiente forma: 108 estudiantes (68,4\%) "tomaría precauciones para salvar su vida"; 36 estudiantes $(22,8 \%)$ "dejaría su vida/muerte en manos del azar (por ejemplo, cruzar descuidadamente una calle muy transitada)"; y finalmente, $14(8,9 \%)$ respondieron "evitaría los pasos necesarios para salvar o mantener su vida (por ejemplo, un diabético que deja de tomar insulina)".

En cuanto a la dimensión temporal - duración, 129 estudiantes $(81,6 \%)$ presentaron pensamientos/conductas suicidas por "períodos breves, pasajeros"; 20 estudiantes (12,7\%) durante "períodos más largos"; y, por último, 9 (5,7\%) de forma "continua (crónica) o casi continua". Con relación a la frecuencia del pensamiento suicida, 98 estudiantes (62\%) presentaron pensamientos/conductas suicidas de forma ocasional/rara; 53 estudiantes $(33,5 \%)$ de forma intermitente; $y$, por último, 7 (4,5\%) de forma persistente/continua. La actitud hacia el pensamiento/deseo fue expresada de la siguiente manera: 71 estudiantes $(44,9 \%)$ contestaron "Ambivalencia, indiferencia"; 69 estudiantes (43,6\%) marcaron la opción "Rechazo"; y, por último, $18(11,5 \%)$ respondieron "Aceptación". Con respecto al control sobre la acción del suicidio o el deseo de llevarlo a cabo, 139 (88\%) encuestados refirieron tener "sensación de control"; 14 $(8,8 \%)$ encuestados "no tuvieron seguridad de control"; y 5 (3,2\%) "no tuvieron sensación de control".

Los factores disuasorios del intento activo fueron en su mayoría la familia $(47,47 \%)$; en segundo lugar, fue la posibilidad de quedar seriamente afectado si fracasara el intento $(16,46 \%)$; en tercer lugar, la irreversibilidad (14,56\%); y después, la religión (8,86\%). El 12,66\% restante mencionó otras causas. Con referencia a las "Razones del proyecto de intento", 138 de los encuestados (87,34\%) contestaron "escapar, acabar, resolver problemas"; $8(5,06 \%)$ dijeron para manipular el medio, atraer atención, venganza; $y$, por último, 12 encuestados (7,59\%) respondieron para una "combinación de ambas". 207 estudiantes $(67,6 \%)$ no intentarían el suicidio debido a algún factor disuasorio; 38 estudiantes $(24,1 \%)$ piensan que los factores disuasorios tienen cierta influencia; $y$, por último, 13 estudiantes (8,3\%) afirman una influencia mínima de los factores disuasorios.

En cuanto a "Método: especificación / planes": 81 de los encuestados respondió que "no los ha considerado"; por otra parte, 63 encuestados respondieron que "los ha considerado, pero sin detalles específicos"; $y$, por último, 14 encuestados mencionó que "los detalles están bien especificados / bien formulados". En cuanto al "Método: accesibilidad / oportunidad": 65 encuestados refirieron un "método no accesible: no hay oportunidad"; 42 encuestados respondieron que "el método llevaría tiempo / esfuerzo; por ende, la oportunidad no es fácilmente accesible"; 40 encuestados respondieron que "tanto método y oportunidad son fácilmente accesibles" $y$, por último, 11 encuestados respondieron "oportunidades o accesibilidad futura del método proyectado". Al preguntar a los estudiantes qué tan capaces se sentían para llevar a cabo un intento de suicidio, las respuestas fueron las siguientes: 98 estudiantes (62\%) se veían a sí mismos como demasiado débiles o sin coraje para llevar a cabo tal acción; 45 estudiantes $(28,5 \%)$ estaban inseguros de su coraje, pero se creían competentes para llevarlo a 
cabo; y 15 estudiantes $(9,5 \%)$ estaban seguros de su competencia y de su coraje para llevar a cabo el suicidio.

Con respecto a la pregunta de si era posible que intentaran suicidarse en un futuro cercano (intento real) la mayoría (108 estudiantes, 68,4\%) respondió que no; 44 estudiantes $(27,8 \%)$ respondieron que no estaban seguros, que era incierto; y una minoría de 6 estudiantes $(3,8 \%)$ respondió que sí. Cuando fueron preguntados sobre su preparación real ante la idea del suicidio, los estudiantes respondieron de la siguiente forma: 133 estudiantes (84,2\%) no hicieron ninguna preparación; 14 estudiantes $(8,9 \%)$ tuvieron una preparación parcial (por ejemplo, empezar a recoger píldoras); y 11 estudiantes (7\%) estaban completamente preparados (por ejemplo, tienen las píldoras suficientes, tienen la navaja, tienen la pistola cargada). En cuanto a la pregunta de si escribió o no alguna nota acerca del suicidio: una mayoría de 133 estudiantes $(84,2 \%)$ respondió que no escribió ninguna nota; 17 estudiantes $(10,8 \%)$ comenzaron a redactarla, pero no la finalizaron y 8 estudiantes $(5,1 \%)$ completaron su carta de suicidio.

Con respecto a los preparativos finales ante la anticipación de la muerte (ejemplo: seguro, testamento, donaciones): 124 estudiantes (78,5\%) respondieron que no realizaron ninguna preparación; 27 estudiantes $(17,1 \%)$ pensaron en dejar algunos asuntos arreglados y una minoría de 7 estudiantes (7,7\%) hizo planes definitivos o dejó todos los asuntos arreglados. Teniendo en cuenta el grado de encubrimiento de los estudiantes hacia las ideas suicidas, la mayoría (43,7\%, 69 estudiantes) mencionó que fue reacio a revelarlas; en contraste un 33,5\% (53 estudiantes) dijo que reveló estas ideas abiertamente. Finalmente, una minoría de 22,8\% (36 estudiantes) admitió que intentó engañar, encubrir o mentir acerca de estas ideas. Con respecto al número de intentos previos de suicidios: la mayoría (84,2\%, 133 estudiantes) respondió que no tuvo ningún intento de suicidio en la vida. En segundo lugar, se situaron 17 estudiantes $(10,8 \%)$ que respondieron que tuvieron un intento de suicidio en la vida. Finalmente, 8 estudiantes $(5,1 \%)$ respondieron que tuvieron más de un intento de suicidio en la vida. Al preguntar a los encuestados cuál fue la intención de morir asociada al último intento, 126 estudiantes (79,9\%) marcaron la opción "Baja"; 23 estudiantes $(14,6 \%)$ marcaron la opción "Moderada, ambivalente, insegura"; y 9 estudiantes (5,7\%) marcaron la opción "Alta".

\section{DISCUSIÓN}

El suicidio es un fenómeno social que involucra a todos los países del mundo; constituye, de acuerdo a estadísticas de la OMS, la segunda causa de muerte en individuos entre los 15 y 29 años de edad y es responsable de al menos 800000 muertes al año (18). Es un tema sumamente delicado e importante y más aun atendiendo a recientes eventos de estas características ocurridos en el Hospital Escuela que sirve a la Facultad de Ciencias Médicas de la Universidad Nacional de Asunción. Esto pone en evidencia el gran estrés al que están sometidos tanto estudiantes como médicos y que puede tener un desenlace trágico. Importante es recalcar que no ocurre solo en nuestro medio, sino que también en el resto del mundo.

La conducta suicida es un problema de salud pública cada vez más frecuente en el ámbito universitario y más aún en personales de la salud. Puede tener diversas causas y es muy importante identificar los rasgos suicidas en las personas en riesgo y, más aún, a la antesala de este suceso: la ideación suicida. Los resultados obtenidos pretenden mostrar la intensidad de los deseos suicidas en los entrevistados, lo que podría alertarnos sobre intentos suicidas a ser realizados. 
El promedio de edades de los participantes fue de 22,79 \pm 2 , con mayor participación en el estudio del sexo femenino $(67,7 \%)$. Estos datos se constatan de manera similar en estudios acerca de ideación suicida llevados a cabo: uno, en estudiantes de Medicina de Pakistán; donde la población de estudio tenía una media de edad de 20 años y mayoritariamente era del sexo femenino; y otro realizado en estudiantes de Medicina de la ciudad de Bucaramanga en Colombia donde las características de la población de estudio eran similares $(19,20)$.

Los resultados muestran que el $54,9 \%$ de la población estudiada tuvo ideas suicidas en su vida y la discusión se centra en este subgrupo a partir de este punto. En un estudio similar, realizado en estudiantes universitarios de la ciudad de México, se encontró que el $18 \%$ ( $22 ; n=122)$ fueron diagnosticados con ideación suicida por la escala ISB y a través de la clínica (16). En otro, realizado en estudiantes de la Universidad Nacional de Colombia, el 4,5\% (5; $n=110)$ tenían ideación suicida actual y el $20 \%(22 ; n=110)$ tuvo ideación suicida por más de una semana durante el último año (7).

Del total de participantes que afirmó tener al menos una idea suicida a lo largo de su vida, el mayor porcentaje correspondió al sexo femenino (65\%), de la misma manera el estudio realizado por Pereira y Cardoso en estudiantes universitarios de Portugal detalla que el $74,4 \%$ de personas con ideación suicida del estudio corresponden al sexo femenino (21). El estudio realizado en Pakistán en estudiantes de Medicina describe que 64,4\% de los estudiantes con ideación suicida pertenecían al sexo femenino (19).

De acuerdo con el deseo de morir o de vivir se vio que una mayoría ha respondido que su deseo de vivir es moderado a intenso $(77,8 \%)$ en contraste con una minoría que ha respondido que su deseo de morir es moderado a intenso (18,4\%). En la intención de morir asociada al último intento de suicidio se encontró que el $9 \%$ presentaba una alta intención de acabar con su vida. En un estudio realizado en estudiantes de colegios y universidades de Colombia, se ha encontrado que los pensamientos acerca de la vida o la muerte en el último/único intento de suicidio, la mayoría de las personas $(42 \% ; n=39)$ consideraban que daba igual vivir que morir, sin embargo, casi $40 \%$ de las personas sí tenían una intención clara de perder la vida, frente a $18 \%(n=39)$ que realmente no lo querían así (22).

En cuanto a las ideaciones con plan suicida, en el presente trabajo se vio que el 8,9 \% ya tenía un plan preestablecido para autoeliminarse, mientras que en un estudio hecho en estudiantes universitarios de Santiago que acudieron a consultas médicas de un servicio estudiantil, hubo un $1,3 \%$ de ideaciones suicidas con plan para realizarlo (6).

Con respecto a la efectividad del método a utilizar para realizar el acto suicida, encontramos que la mayoría dudaba de tal efectividad, pues el $41,1 \%$ ha respondido que el método no estaba disponible, es decir, no había oportunidad de morir con tal acto; a diferencia de un 7\% que estaba seguro de la accesibilidad y oportunidad futura del método elegido. En el estudio ya citado anteriormente (22), la mayoría (59,5\%) de las personas tenían dudas también sobre la efectividad del método que iban a utilizar, ya que no tenían claro si vivirían o morirían, mientras que tan sólo un 33\% de los casos esperaban la muerte como un evento seguro.

En nuestro estudio el $15,8 \%$ de los estudiantes con ideación suicida tuvieron por lo menos un intento de suicidio, lo que es muy superior a otros estudios como el realizado en estudiantes 
de la Universidad de Guanajuato (México) en donde se vio un $8,6 \%$ de intentos suicidas. Asimismo, nuestros resultados contrastan con el $6,3 \%$ de estudiantes universitarios con intento de suicidio de Santiago ya mencionado, y que a su vez son superiores a los observados en estudiantes menores de 18 años de la Universidad Nacional de Colombia, sede Bogotá, con un $0,03 \%$ y a los obtenidos en otro trabajo realizado en la misma Universidad por otros autores en el que ningún estudiante tuvo intentos suicidas, pero con la salvedad de que en estas dos últimas investigaciones solo se valoró esa posibilidad en el último año de sus vidas $(6,7,23$, 24). También se debe tener en cuenta que los datos mencionados anteriormente corresponden a comparaciones con universitarios de diversas carreras. Un trabajo hecho en estudiantes de Medicina de la Universidad de Bucaramanga en Colombia reveló que el 5\% ya había realizado por lo menos un intento suicida (20).

Como elemento disuasorio del intento activo de suicidio, la familia como elemento de apoyo se presentó con mayor frecuencia (47\%). Resulta interesante este dato al contrastar lo hallado por el estudio en estudiantes de Medicina pakistaníes, donde se determinó una relación significativa entre frecuencia de ideación e intento suicida y negligencia por parte de la familia. Los participantes que referían sentir menos apoyo por parte de sus familias presentaban mayor porcentaje de intento suicida (19).

Se puede afirmar que la ideación suicida es un fenómeno de frecuente ocurrencia en estudiantes universitarios. Es necesario que las Universidades establezcan programas de detección e intervención temprana que permitan disminuir la probabilidad de ocurrencia de desenlaces negativos.

Es evidente que el número de estudios realizados acerca de la ideación suicida en facultades de Medicina en Paraguay es limitado, motivo por el cual sería conveniente realizar estudios en muestras grandes, especialmente comparativas con otras carreras, para determinar si los estudiantes de Medicina tienen un perfil psicológico suicida distinto a los estudiantes de otras carreras.

\section{Conclusiones}

La frecuencia de ideación suicida en los estudiantes de Medicina de la Facultad de Ciencias Médicas de la Universidad Nacional de Asunción en el año 2017 fue del 54,9\%.

Entre los estudiantes con ideación suicida, la intensidad del deseo de vivir fue en su mayoría de moderado a intenso representado por un $77,2 \%$. Entretanto, la intensidad del deseo de morir fue mayoritariamente débil con un $51,9 \%$. En cuanto a la duración y frecuencia de los pensamientos/comportamientos suicidas, se encontró que en su mayoría se presentaron de forma ocasional, rara $(62,02 \%)$ y por períodos breves y pasajeros $(81,6 \%)$. La razón más frecuente del proyecto de intento suicida fue la de escapar, acabar y resolver problemas $(87,34 \%)$. Con respecto a los factores disuasorios del intento de suicidio, se registró que el factor más frecuente, con un $47 \%$, está representado por la familia del estudiante.

Con los resultados obtenidos se propone la necesidad de inclusión de programas de prevención, detección y abordaje de dificultades en salud mental en la Facultad de Medicina donde se realizó el presente trabajo. La ideación suicida es un fenómeno de frecuente ocurrencia en estudiantes de Medicina. Es necesario que directivos, docentes y toda la comunidad educativa de la Facultad de Ciencias Médicas de la Universidad Nacional de Asunción aúnen esfuerzos 
en la concreción de programas de detección e intervención temprana que permitan disminuir la probabilidad de ocurrencia de desenlaces negativos.

\section{CONFLICTOS DE INTERÉS Y FUENTE DE FINANCIACIÓN}

Los autores declaran no poseer conflictos de interés. Fuente de financiación: ninguna.

\section{REFERENCIAS BIBLIOGRÁFICAS}

1. Organización Mundial de la Salud [Internet]. Who.int; c2016 [cited 10 Oct 2017]. Prevención del suicidio, recurso para consejeros [approx. 18 screens]. Available from: http://www.who.int/mental health/media/counsellors spanish.pdf

2. Moscicki E. Epidemiology of Suicidal Behavior. Suicide Life Threat Behav. 1995;25(1):22-35. URL.

3. Rice DP, Miller LS: The economic burden of affective disorders. Br J Psychiatry Suppl. 1995;(27):34-42. URL.

4. Denis-Rodríguez E, Barradas Alarcón M, Delgadillo-Castillo R, Denis-Rodríguez P, MeloSantiesteban G. Prevalencia de la ideación suicida en estudiantes de Medicina en Latinoamérica: un meta análisis. Ride. 2017;8(15):1-32. https://doi.org/10.23913/ride.v8i15.304

5. Maris RW. Suicide and life threatening behavior. Introduction. Suicide Life Threat Behav. 1991;21(1):1-17. URL.

6. Micin S, Bagladi V. Salud Mental en Estudiantes Universitarios: Incidencia de Psicopatología y Antecedentes de Conducta Suicida en Población que Acude a un Servicio de Salud Estudiantil. Ter Psicol. 2011;29(1): 53-64. https://doi.org/10.4067/S0718$\underline{48082011000100006}$

7. Calvo JM, Sánchez R, Tejada PA. Prevalencia y Factores Asociados a Ideación Suicida en Estudiantes Universitarios. Rev. salud pública 2003;5(2): 123-143. URL.

8. Mann J, Oquendo N, Underwood M, Arango W. The neurobiology of suicide risk: a review for de clinician. J Clin Psychiatry. 1999; 60(Suppl 2): 7-11. URL.

9. Mortensen P, Agerbo E, Erikson T, Qin P, Westergaard-Nielsen N. Psychiatric illness and risk factors for suicide in Denmark. Lancet 2000;355(9197):9-12. URL.

10. Powell J, Geddes J, Deeks J, Goldcare M, Hawton K. Suicide in psychiatric hospital inpatients. Risk factors and their predictive power. Br J Psychiatry. 2000;176:266-272. URL.

11. Cova Solar F, Alvial W, Aro M, Bonifetti A, Hernández M, Rodríguez C. Problemas de Salud Mental en Estudiantes de la Universidad de Concepción. Ter Psicol. 2007;25(2): 105-112. https://doi.org/10.4067/S0718-48082007000200001

12. Sánchez Sosa JC, Villarreal González ME, Musitu G, Martínez Ferrer B. Ideación Suicida en Adolescentes: Un Análisis Psicosocial. Psychosocial Intervention. 2010;19(3):279287. URL.

13. Adorno SC, Pagliaro NR. Situación actual del suicidio y tentativa de suicidio en el Paraguay. An. Fac. Cienc. Méd. (Asunción) 2012;45(2):25-38. URL.

14. Barrios I, Miltos V, Piris A, Piris G, Ramírez C, Rodríguez J, Torales J, et al. Tamizaje de salud mental mediante el test MINI en estudiantes del ciclo básico de Medicina de la Universidad Nacional de Asunción. An. Fac. Cien. Méd. (Asunción) 2015;48(1):59-66. https://doi.org/10.18004/anales/2015.048(01)59-068 
15. Melipillan Araneda R, Cova Solar F, Rincón González P, Valdivia Peralta M. Propiedades Psicométricas del Inventario de Depresión de Beck-II en Adolescentes Chilenos. Ter Psicol. 2008;26(1):59-69. https://doi.org/10.4067/S0718-48082008000100005

16. González Macip S, Díaz Martínez A, Ortiz León S, González C, González Núñez JdJ. Características psicométricas de la Escala de Ideación Suicida de Beck (ISB) en estudiantes universitarios de la ciudad de México. Salud Mental 2000; 23(2):21-30. URL.

17. Beck AT, Kovacs M, Weissman A. Assessment of suicidal intention: the Scale for Suicide Ideation. J Consult Clin Psychol. 1979;47(2):343-352. URL.

18. Organización Mundial de la Salud [Internet]. Who.int; c2017 [cited 10 Oct 2017]. Suicidio [approx. 2 screens]. Available from: http://www.who.int/topics/suicide/es/

19. Osama M, Islam MY, Hussain SA, Masroor SM, Burney MU, Masood MA, et al. Suicidal ideation among medical students of Pakistan: A cross-sectional study. J Forensic Leg Med. 2014;27:65-68. https://doi.org/10.1016/i.jflm.2014.08.006

20. Pinzón-Amado A, Guerrero S, Moreno K, Landínez C, Pinzón J. Ideación suicida en estudiantes de medicina: prevalencia y factores asociados. Revista Colombiana de Psiquiatría 2013;43(1):47-55. https://doi.org/10.1016/j.rcp.2013.11.005

21. Pereira A, Cardoso F. Suicidal Ideation in University Students: Prevalence and Association With School and Gender. Paidéia (Ribeirão Preto) 2015;25(62):299-306. https://doi.org/10.1590/1982-43272562201503

22. Villalobos-Galvis FH. Situación de la conducta suicida en estudiantes de colegios y universidades de San Juan de Pasto, Colombia. Salud Ment. 2009;32(2): 165-171. URL.

23. Chávez Hernández AM, Pérez Hernández R, Macías García LF, Páramo Castillo D. Ideación e intento suicida en estudiantes de nivel medio superior de la Universidad de Guanajuato. Acta universitaria 2004;14(3):12-20. https://doi.org/10.15174/au.2004.221

24. Sánchez R, Cáceres H, Gómez D. Ideación suicida en adolescentes universitarios: prevalencia y factores asociados. Biomédica 2002;22(Su2):407-416. URL. 